\title{
Genetic Characterization of Pitahaya Accessions Based on Amplified Fragment Length Polymorphism Analysis
}

\author{
Deborah Pagliaccia ${ }^{1}$, Georgios Vidalakis, and Greg W. Douhan ${ }^{2}$ \\ Department of Plant Pathology and Microbiology, University of California, \\ 900 University Avenue, Riverside, CA 92521
}

\author{
Ramiro Lobo ${ }^{1,2}$ and Gary Tanizaki \\ University of California, Cooperative Extension, San Diego County, San \\ Diego, CA 92123
}

Additional index words. genetic diversity, germplasm, AFLP, Dragon fruit

\begin{abstract}
Hylocereus (Berger) Britton and Rose and Selenicereus (Berger) Britton and Rose are two genera of vine cacti, commonly named pitahaya, that produce fruit that are gaining in popularity as an exotic fruit in many countries. There has also been an increasing interest in these fruits because they can be grown in areas that are prone to drought and heat where other fruit production is not possible. However, there is significant taxonomic confusion regarding species within these genera as well as some uncertainty among named varieties. Amplified fragment length polymorphism (AFLP) was used to genotype a large pitahaya collection to determine if there was redundancy in some of the commonly named varieties as well as to assess the overall diversity of the collection. A total of 51 markers were scored from 230 accessions. Seven distinct clades were found with 126 putative clones but only three clades had high bootstrap (greater than $80 \%$ ) support and one had moderate support $(60 \%)$. Some of the differently named varieties were identical based on our analysis, but there was also genotypic diversity within putatively named varieties. The results of this study will help growers and researchers to choose genetically distinct accessions from the germplasm collection to investigate how different accessions perform in their growing regions.
\end{abstract}

Dragon fruit, strawberry pear, pitahaya, and pitaya are all common names for the fruit of vine cacti from species of Hylocereus (Berger) Britton and Rose and Selenicereus (Berger) Britton and Rose. For the purposes of this article, we refer to all of them collectively as pitahaya. These cacti are native to northern South America, Central America, and Mexico but have been propagated and distributed to many countries for agricultural production (Lobo, 2007). They are commercially being grown in the United States (Crane and Balerdi, 2009; Merten, 2003; Thomson, 2002; Valdivia, 2000), Central and South America (Instituto Nicaraguense de Reforma Agraria, 1994; Lobo, 2007), Asia (Komang, 2010; Raveh et al., 1993), Africa (Joubert, 2012), and Australia (Dimitri, 1999; Luders and McMahon, 2006). There has been an increasing interest in these

Received for publication 15 Oct. 2014. Accepted for publication 9 Jan. 2015.

We acknowledge the University of California Division of Agriculture and Natural Resources and the University of California Thelma Hansen Agricultural Research Fund for the financial support to establish the germplasm collection and to conduct this study.

${ }^{1}$ Co-first authors.

${ }^{2}$ To whom reprint requests should be addressed; e-mailgdouhan@ucr.edu or relobo@ucanr.edu. fruits because they can be grown in areas that are prone to drought and heat where other fruit production is not possible (Nobel and De LaBarrera, 2004; Raveh et al., 1998). Moreover, this fruit has gained more attention in recent years as a result of the increasing demand for new and exotic fruits, which makes the look and attractive colors of the skin and flesh of pitahaya very appealing to consumers. Pitahaya are full of essential nutrients such as complex carbohydrates, minerals, vitamins, fibers, and antioxidants, which may also explain their increase in demand for health purposes (LeBellec et al., 2006). In fact, current demand and market prices indicate that consumer preference is shifting away from white-fleshed varieties in favor of colored-flesh varieties (pink or red) because they have more complex flavors and are perceived to have higher nutritional value.

The four most common species commercially grown are $H$. undatus, $H$. polyrhizus, $H$. costaricensis, and $S$. magalanthus (syn. H. malanthus) (Ortiz and Carrillo, 2012). With the exception of southeast Asia, the cultivation of each of these species follows their geographic origin. As a result, $H$. polyrhizus and $H$. costaricensis have been cultivated in Central America (Nicaragua); $H$. megalanthus has been primarily cultivated in South America (Colombia and Ecuador); H. undatus has been primarily cultivated in southeast Asia (Vietnam, Thailand, Taiwan, and the Phillipines) and some areas of southern Mexico (Castillo-Martinez et al., 2005). However, pitahaya species hybridize quite easily and since the late 1980s and early $1990 \mathrm{~s}$ amateur breeders in the United States, Israel, and southeast Asia have developed a number of hybrids (Thomson, 2002). These hybrids generally include crosses between either $H$. guatemalensis or $H$. megalanthus with $H$. undatus as the other parent. These purposeful hybridizations have resulted in plants with great adaptability to the local environment and which produce fruit of exceptional quality. Many of these hybrids are available as commercial varieties and have become quite popular among growers in the United States (i.e., 'Physical Graffiti', 'Halley's Comet', 'Delight', etc.). Recent efforts by private breeders in the United States and Israel have also resulted in newer hybrids, which are also commercially available as proprietary varieties, but the adoption of these has been limited (Lobo, personal communication).

There is considerable taxonomic confusion surrounding these cacti. In addition to the relative ease of hybridization, pitahaya is easily propagated by cuttings, which make it a popular and commonly exchanged plant among backyard growers in the United States. Superior clones or hybrids are distributed quite rapidly and in many cases individual growers will assign their own names to previously named varieties, thus creating duplicate names and exacerbating the taxonomic confusion. Botanists have added to the confusion as well. This is the case with $H$. magalanthus, which was originally placed in a newly erected genus, Mediocactus (Britton and Rose, 1920), because it appeared to be morphologically intermediate between Selenicereus and Hylocereus. Subsequently, the species was transferred to the genus Selenicereus by Morgan (1953) but was later treated as Hylocereus by Bauer (2003). This intermediate morphology, along with the finding that $H$. magalanthus has double the number of chromosomes found in related species $(2 n=44$ vs. $2 n=22)$, led to the hypothesis that this species was in fact a polyploid and potentially a hybrid between unknown species from the two genera (e.g., Plume et al., 2013).

This taxonomic confusion is also exacerbated by the fact that intraspecies/intravarietal morphological differences often occur, likely as a result of environmental conditions (Castillo-Martinez et al., 2005), which makes erecting and evaluating "new" varieties or cultivars extremely difficult. Collectively, this led to the International Union for the Protection of New Varieties of Plants (UPOV, 2011) to propose a guideline to document how new varieties of pitahaya are established. However, as previously mentioned, intraspecies/ intravarietal morphological differences among vegetative clones and hybridization within this group leads to genetic mosaics among new lines and makes identification among varieties extremely difficult. A more practical and less subjective approach is to use molecular 
markers to differentiate among the various species and/or cultivars, but this approach has only been attempted in three studies of pitahaya based on limited sampling (Legaria Solano et al., 2005; Tao et al., 2014; Tel-Zur et al., 2004).

A large collection of pitahaya germplasm accessions are curated at the University of California South Coast Research and Extension Center (SCREC), Irvine, CA. The collection was first established in 2005 with the goal of evaluating pitahaya varieties for commercial production in southern California. It started with seven varieties originating from Nicaragua including an estimated 120 individual plants representing four different species (H. undatus, $H$. polyrhizus, $H$. costaricensis, and unnamed Hylocereus sp.); two varieties originating from Mexico included 34 individual plants representing two species ( $H$. ocamponis and $H$. megalanthus); two varieties sourced from San Diego and included 34 individual plants representing two species (H. undatus and H. guatemalensis); and seven varieties sourced from Florida including 120 individual plants representing four species and hybrids (H. undatus, $H$. guatemalensis, $H$. megalanthus, and a number of putative hybrids identified as Hylocereus spp.) Additional accessions have been intermittently added to the collection, including 17 additional accessions from Nicaragua (within the $H$. polyrhizus/costaricensis group). These accessions were tentatively labeled as $H$. costaricensis/polyrhizus because both of these species are commonly found in Nicaragua and throughout Central America but the distinction between them is not clearly determined or easily established. One additional variety from Mexico (17 individual plants representing $H$. ocamponis) was acquired and several other varieties were also sourced from Florida. Additional material has been sourced from local producers and two plants per accession have been added to the collection. In total, the collection now includes 378 individual plants, representing potentially 54 varieties and seven different species.

However, as the collection was being established, the close examination of morphological traits among the various accessions indicated significant similarities among plants from different varieties and significant differences among plants within the same varieties (Lobo, personal observation). This suggested that some plants within each of the designated varieties were different and that some of the designated cultivars with different names may indeed be the same clone. Therefore, the objectives of this work were to use molecular data to genotype a large number of plants in the collection to test this hypothesis and to obtain preliminary data regarding how much genetic diversity is present within the collection.

\section{Materials and Methods}

Sample collection and DNA isolation. Pitahaya plant specimens were sampled in Oct. 2012 from SCREC. Plant material was collected by cutting $\approx 10$-cm segments from the tips of actively growing shoots and transporting them on ice to the laboratory. The samples where then stored at $4{ }^{\circ} \mathrm{C}$ until processed for DNA extraction.

The plant material was surface-sterilized by dipping the stem into $75 \% \mathrm{EtOH}$ for 15 to $30 \mathrm{~s}$ and rinsing with sterile water. Sterile blades were then used to slice off the margins of the stem edge in an effort to avoid cutting too deep into the tissue to minimize the amount of inner mucilaginous material as much as possible. The material was then cut into 0.3 - to $0.5-\mathrm{mm}$ pieces and two $2-\mathrm{mL}$ centrifuge tubes were filled and stored at $-80{ }^{\circ} \mathrm{C}$ until freeze-dried. The material was freeze-dried using a FreeZone Triad Cascade Freeze Dry System (Labonco, Kansas City, MO) for $15 \mathrm{~h}$ at $-25{ }^{\circ} \mathrm{C}$ and $7.5 \mathrm{~h}$ at $10{ }^{\circ} \mathrm{C}$ using a freeze-drying and vacuum-drying system and stored at $-80{ }^{\circ} \mathrm{C}$ until further processing. To macerate the tissue, two sterile $5 / 32$-inch stainless steel grinding balls were placed in each tube and ground for 20 to $30 \mathrm{~s}$ using a Geno Grinder 2010 Spex Sample Prep instrument (SPEX SamplePrep, Metuchen, NJ).

For extraction of DNA, $25 \mathrm{mg}$ of lyophilized tissue was transferred to a $1.5-\mathrm{mL}$ tube. The extra tissue was kept frozen at $-80{ }^{\circ} \mathrm{C}$ for later use. DNA was isolated using DNeasy Plant Mini Kits (Quiagen Inc., Valencia, CA) with minor changes to the manufacturer instructions. Briefly, cells were lysed by adding $700 \mu \mathrm{L}$ lysis buffer and incubated at $65^{\circ} \mathrm{C}$ for $10 \mathrm{~min}$. Before the incubation step, $4 \mu \mathrm{L}$ of RNase was added to the lysate, then one 5/32-inch stainless steel grinding ball was placed in each samples tube and vortexed at $850 \mathrm{rpm}$ for $40 \mathrm{~s}$ with the Geno Grinder 2010 to remove any clumps. After lysis, proteins and polysaccharides were salt-precipitated. Cell debris and precipitates were removed by a 30-min spin at room temperature at 14,000 rpm through QIAshredder spin columns (Qiagen Inc., Valencia, CA). The cleared lysate was transferred to a new tube and binding buffer and ethanol were added to bind the DNA to the DNeasy membrane. The sample was then applied to a DNeasy spin column and spun briefly in a centrifuge. Contaminants (proteins and polysaccharides) were removed by two wash steps and then the DNA bonded to the membrane was eluted in a $70-\mu \mathrm{L}$ volume of low-salt buffer $\mathrm{AE}$. The quality and quantity was evaluated in $0.8 \%$ agarose gels stained with SYBR Green I (Molecular Probes, Eugene, OR).

Amplified fragment length polymorphisms. The method of Vos et al. (1995) was used to develop the initial restriction-ligation products used for a pre-amplification polymerase chain reaction (PCR) amplification, but fluorescently labeled primers were used to resolve the markers through a capillary sequencer in the secondary amplification step as described below.

A pre-amplification PCR of the initial restriction-ligation products was performed using randomly chosen primer combinations with the addition of one selective nucleotide at the 3' ends of the ECORI and MSEI specific primers. For the PCR pre-amplification, each $20-\mu \mathrm{L}$ reaction contained $1 \times$ PCR buffer
(Invitrogen, Carlsbad, CA), $2.5 \mathrm{~mm} \mathrm{MgCl2}$, $2.5 \mathrm{~mm}$ each dNTP (Invitrogen), $0.375 \mu \mathrm{M}$ of each primer, $0.5 \mathrm{U}$ of Taq polymerase (Invitrogen), and $10 \mu \mathrm{L}$ of template of the restriction ligation dilution. Thermocycling conditions consisted of an initial hold at $95{ }^{\circ} \mathrm{C}$ for $4 \mathrm{~min}$, followed by 20 cycles with a denaturation step of $94{ }^{\circ} \mathrm{C}$ for $30 \mathrm{~s}$, an annealing temperature of $56^{\circ} \mathrm{C}$ for $1 \mathrm{~min}$, and an extension temperature of $72{ }^{\circ} \mathrm{C}$ for $1 \mathrm{~min}$ followed by a final elongation step at $72{ }^{\circ} \mathrm{C}$ for $5 \mathrm{~min}$. All amplifications were performed in a MyCycler (Bio-Rad Laboratories Inc., Hercules, CA).

For the second AFLP amplification, a total of 14 randomly chosen primer combinations with the addition of two or three selective nucleotides at the $3^{\prime}$ end were used to screen a subset of eight samples and four primer combinations were chosen for the final analysis, which resolved the fragment patterns well under the experimental conditions used. The four chosen primer combinations had the sequences for the ECORI $(\mathrm{E}+1)$ and MSEI $(\mathrm{M}+1)$ adapters followed by the addition of FAM ECO RI +GAT/MSE I +CA, FAM ECO RI +GC/MSE I +GAC, HEX ECO RI + GCT/MSE I +CA, and HEX ECO RI $+\mathrm{GCT} / \mathrm{MSE} \mathrm{I}+\mathrm{GT}$ at the $3^{\prime}$ prime ends. For AFLP amplification, each $20-\mu \mathrm{L}$ reaction contained $1 \times$ PCR buffer (Invitrogen), $2.5 \mathrm{~mm}$ $\mathrm{MgCl} 2,2.5 \mathrm{~mm}$ each dNTP (Invitrogen), $0.375 \mu \mathrm{M}$ of each primer, $0.5 \mathrm{U}$ of Taq polymerase (Invitrogen), and $10 \mu \mathrm{L}$ of template of the pre-amplification PCR diluted $1: 10$ or $1: 25$ depending on the PCR product quantity. Thermocycling conditions consisted of an initial hold at $72{ }^{\circ} \mathrm{C}$ and $94{ }^{\circ} \mathrm{C}$ for 1 and $4 \mathrm{~min}$, respectively, followed by 44 cycles with an annealing temperature of $65^{\circ} \mathrm{C}$ for $30 \mathrm{~s}$ and an extension temperature of $72{ }^{\circ} \mathrm{C}$ for $1 \mathrm{~min}$ with the annealing temperature reduced by $1{ }^{\circ} \mathrm{C}$ for the first nine cycles. Twenty accessions were used as controls by conducting independent DNA extractions, restriction-ligation reactions, PCR pre-amplifications, and secondary AFLP-PCR amplifications.

The labeled FAM and HEX fragments were detected using an ABI 3100 16-capillary instrument at the CORE Instrumentation Facility at the University of CaliforniaRiverside. Genographer 2.0 fragment analysis software was used to score the data by setting the intensity settings between 1 and 5 and manually scoring the presence or absence of clearly visible polymorphic bands above $100 \mathrm{bp}$ by checking chromatograms as well as an electronic image of the gel (Benham et al., 1999). Putative alleles at each polymorphic AFLP locus were scored using a binary code $(1,0)$ corresponding to positive and null alleles, respectively. For the selection of AFLP markers, only polymorphic loci that were reproducible and easily identifiable were chosen.

Cluster analyses on genetic distances the mean character difference option and unweighted pair-group method with arithmetic averaging (UPGMA) were conducted using PAUP* (Version 4.0 beta 10) (Swofford, 
2002). Confidence in tree topology was examined using non-parametric bootstrap with 1000 bootstrapped replicates within PAUP.

\section{Results}

A total of 51 polymorphic loci were scored for 230 accessions and the independent controls produced identical results. We detected seven main clades and 126 putative clones that grouped the accessions into potential species, a wild-type accession group and one hybrid group; $H$. guatemalensis, $H$. undatus, hybrids, wild-type accessions, $H$. megalanthus, $H$. costaricensis/polyrhizus, and H. ocamponis (Fig. 1). However, high bootstrap support was only found for the wild-type accession clade (96\%), the H. megalanthus clade (94\%), and the $H$. ocamponis clade $(83 \%)$. Moderate bootstrap support was found for the $H$. guatemalensis clade $(60 \%)$. Table 1 summarizes the number of accessions, unique genotypes, and identical accessions within each clade as well as the putative varieties and their origin, i.e., where these accessions were originally collected.

Within the H. guatemalensis clade, three known varieties ('Bien Hoa Red', 'American Beauty', and 'Halley's Comet') (Table 1) were found with one clonal cluster that contained accessions with differently named varieties ('Bien Hoa Red' and 'American Beauty') (Fig. 1). There were also two accessions that had been originally thought to be $H$. undatus that clustered in this group as well as two putative unnamed hybrid accessions. One accession that was designated as 'Halley's Comet' also clustered in this clade, but the rest of the 'Halley's Comet' accessions all clustered in the hybrid clade. However, one of 'Halley's Comet's' parent is H. guatemalensis, which could explain this (Thomson, 2002). Within the $H$. undatus clade, three named varieties ('Seoul Kitchen', 'Vietnamese Giant', and 'Mexicana') (Table 1) were found with one clonal cluster that contained accessions with differently named varieties ('Mexicana' and 'Vietnamese Giant') (Fig. 1). Multiple unnamed accessions were also identical to several 'Seoul Kitchen' accessions. Within the hybrid group clade, three named varieties ('Delight', 'Halley's Comet', and 'Physical Graffiti') (Table 1) were found with two clonal clusters that contained accessions with differently named varieties ('Delight' and 'Halley's Comet'; 'Physical Graffiti' and 'Halley's Comet') (Fig. 1) but they all share the same parents (Thomson, 2002). Two unnamed accessions from the collection also fell within this clade. Two named varieties ('Sin Espinas' and 'San Ignacio') (Table 1) were found within the wild-type accession clade with no clonal genotypes found with confounding names. Within the $H$. megalanthus clade, two name varieties ('Colombiana' and 'Yellow Dragon') (Table 1) were found with no clonal genotypes identified with confounding names. Within the $H$. costaricensis/polyrhizus clade, six named varieties ('Rosa', 'San Ignacio', 'Cebra', 'Lisa', 'Orejona', 'Armando') (Table 1) were found with two clonal groups that contained accessions with differently named varieties ('San Ignacio' and 'Rosa'; 'Lisa', 'Orejona', 'Cebra', and 'Rosa') (Fig. 1). Multiple clonal accessions were also found within the collection (Table 1; Fig. 1) and five unnamed accessions also fell within this clade. Within the $H$. ocamponis clade, two named varieties ('El Grullo' and 'Valdivia Roja') (Table 1) were found with no clonal genotypes found with confounding names.

\section{Discussion}

This is the first comprehensive genetic analysis of a large collection of pitahaya accessions. As was initially suspected, some of the named varieties were indeed identical based on our analysis of 51 molecular markers. However, even within each clade, there was also genotypic diversity within putatively named varieties. This is not surprising because outcrossing can occur. Moreover, relative ease of propagation has made pitahaya a popular plant among hobby breeders and backyard growers, which has likely also contributed to the wide distribution of plant material. Collectively, this has further contributed to the taxonomic confusion associated with this plant, which in turn has hindered the work of professional breeders. Without the proper identification of the species or varieties, it becomes increasingly difficult to develop new genotypes with desirable characteristics and may also contribute to limit the growth of the pitahaya industry as well.

One of the main objectives of the current study was to determine if multiple accessions were potentially the same clone. It is possible that many cultivars could have been renamed because germplasm has been distributed easily and the pitahaya plants are mostly propagated clonally by cuttings. This is a common practice because it ensures that propagated plants are identical to the mother plant and to shorten the establishment period to expedite fruit production. After several years of collecting and growing this SCREC pitahaya germplasm collection, it was apparent that some named varieties in the collection could potentially be the same as a result of morphological similarities (Lobo, personal observation). One of the first potential cultivars that were putatively the same clone were 'Bien Hoa Red' and 'American Beauty', which belonged to the $H$. guatamalensis clade. The former is an accession from San Diego, CA, and the latter is from Florida. It is rumored that the source material for the variety originating in Florida was actually obtained in San Diego (Sven Merton, personal communication). This situation provides just another example where lack of information and uncertainty with regard to the taxonomy of pitahaya further exacerbates confusion among breeders, extension personnel, and growers.

To the best of our knowledge, only three other studies have used molecular markers to study the genotypic diversity of pitahaya. Tel-Zur et al. (2004) used random amplified polymorphic DNA (RAPD) markers that differentiated 13 species within Hylocereus and Selenicereus. Moreover, Tel-Zur et al. (2004) were able to differentiate unique genotypes between different accessions of $H$. polyrhizus, H. undatus, S. grandiflorus, and $S$. megalanthus ( $H$. megalanthus), which are consistent with the results of the present study. Legaria Solano et al. (2005) also used RAPD markers to evaluate the genetic diversity among 50 accessions of $H$. undatus originating from nine different states in Mexico and one accession from Colombia as a control (unknown accession). In addition to identifying each accession, Legaria Solano et al. (2005) found three genotypes that were significantly different from the rest and demonstrated substantial genetic diversity of pitahaya in Mexico. In a more recent study, Tao et al. (2014) used intersimple sequence repeat markers to differentiate 50 accessions from the Dragon Fruit Germplasm Collection of Guizhou Institute of Fruit Tree Science in China. All of the genotypes tested by Tao et al. (2014) were unique and clustered into three main groups: one consisting of mostly wild accessions, the second consisting of $H$. polyrhizus accessions (red pulp types), and the third consisting of $H$. undatus accessions (white pulp types). However, they also scored 15 morphological traits, which resulted in some discrepancies within the cluster analysis (UPGMA) compared with the molecular data. Their results further emphasize the confusion in the taxonomy of this group based on classical morphology and this is likely because there are no reproductive barriers among most "species" in pitahaya.

Our results also support limited reproductive barriers among most accessions because some accessions that were named as different species clustered into a different main clade than the original identification. However, there was only strong bootstrap support for three of the clades (wild-type accessions, $H$. megalanthus, $H$. ocamponis). This finding also supports the hypothesis that lack of reproductive barriers among most pitahaya "species" leads to genetic mosaics. Similar results have also been found in other highly outcrossing species of plants. For example, in avocado (Persea sp.), three main botanical races are recognized but hybridization among the races is common. Thus, reciprocal monophyly of alleles among species does not occur, which can result in low bootstrap support in molecular analyses. In support of this idea, Ashworth and Clegg (2003) analyzed 33 avocado cultivars based on 25 microsatellite markers but found very little bootstrap support for each of the clades they found. They hypothesized that the weak bootstrap support for clusters of cultivars was the result of using a large number of hybrid cultivars and suggested that hybrids unite alleles from two genetically distinct sources, thereby decreasing the phylogenetic signal.

The results presented in this study have revealed not only the potential misidentifications of many SCREC accessions, but have also shown that of the 230 accessions tested, only 126 putative clones were found. This is 


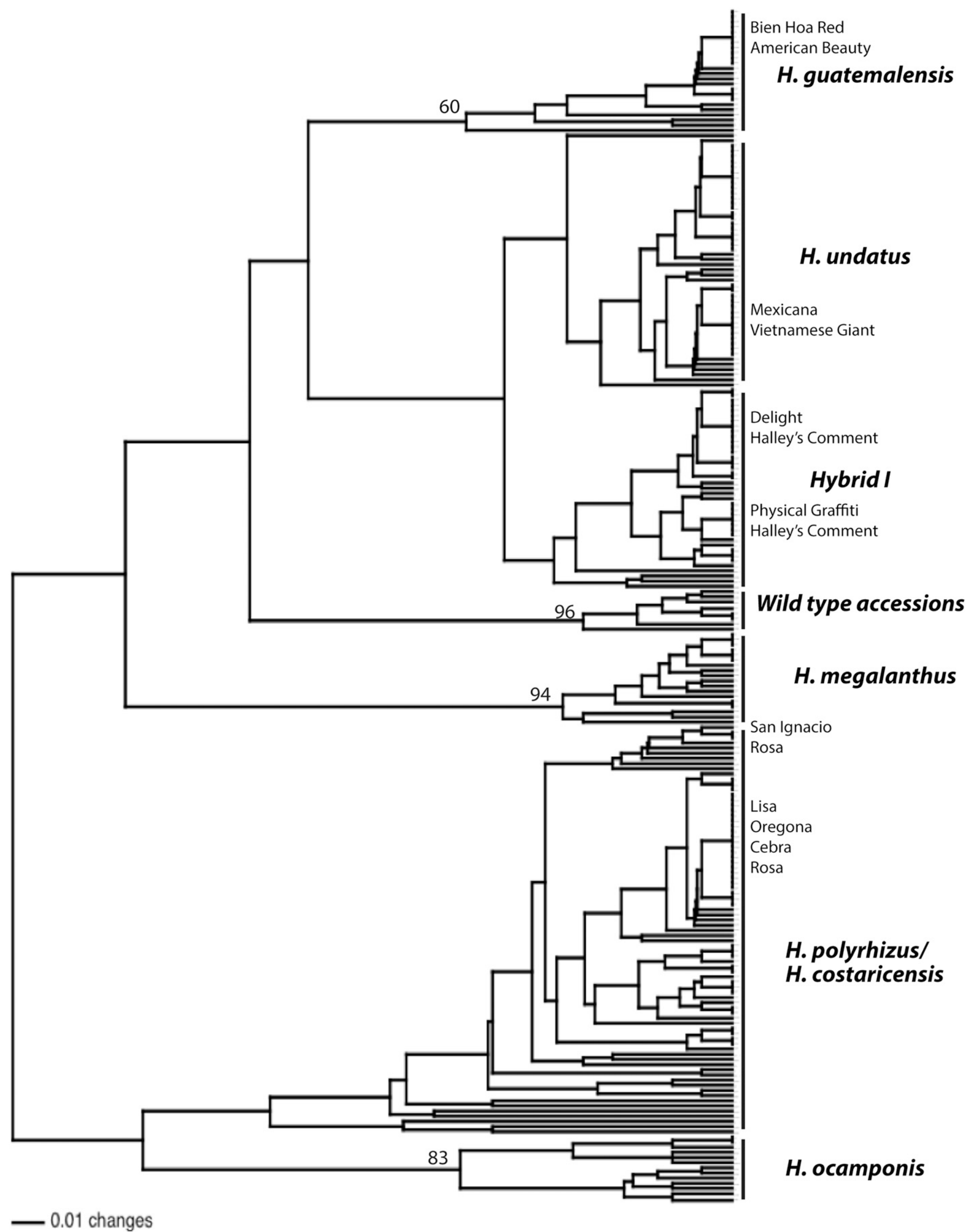

Fig. 1. Dendogram of pitahaya accessions based on unweighted pair-group method with arithmetic averaging (UPMGA) cluster analysis using PAUP* with genetic distances calculated from 51 polymorphic amplified fragment length polymorphism (AFLP) loci. Bootstrap support values above $50 \%$ are shown based on 1000 replicates. The seven main clades are noted as well as putative clonal accessions (straight lines) where conflicts in the originally assigned varietal names were found based on this study from the South Coast Research and Extension Center collection. 
Table 1. Summary of the genotypic results for the Hylocereus/Selenicereus spp. used in this study.

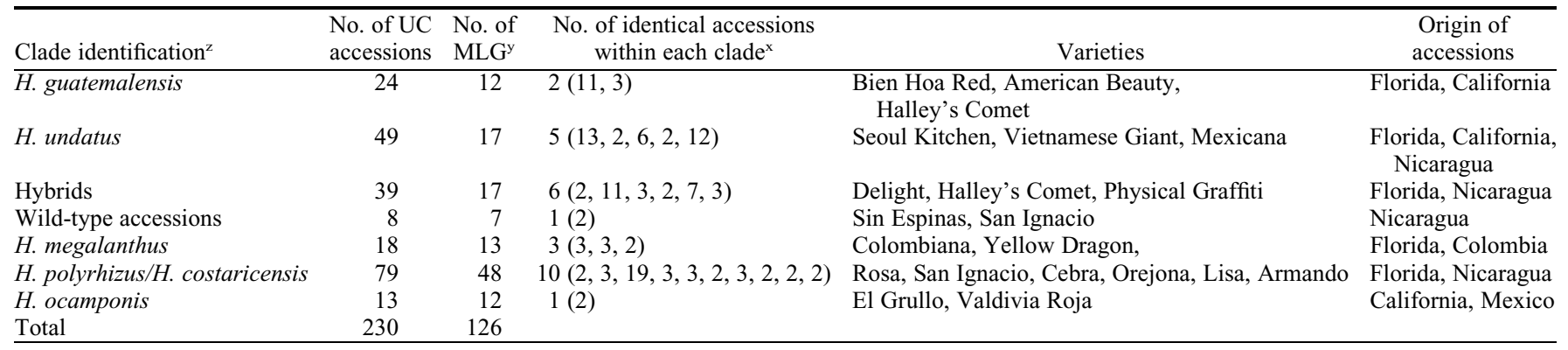

${ }^{2}$ Identification with respect to Figure 1.

${ }^{\mathrm{y}} \mathrm{MLG}=$ multilocus genotypes.

${ }^{\mathrm{x}}$ Numbers in parentheses indicate the number of putative clonal accessions for each cluster of clones within each clade based on Figure 1.

a significant finding because this genetic information can lead to better trials to determine which accessions perform best under California conditions and will reduce redundancy of accessions that are either the same or genetically similar. Thus far, research efforts conducted over the past several years have demonstrated that pitahaya adapts well to field-growing conditions in coastal southern California counties, from San Luis Obispo to San Diego County, and could potentially be a crop that is economically valuable to California growers. Interest among growers has increased, particularly small-scale producers looking for alternatives to replace Valencia oranges and avocado groves. Pitahaya acreage has also increased, primarily because of the significant increase in water costs making this drought tolerance crop more economically attractive. For example, pitahaya acreage has increased from approximately zero commercial acres in the early 1990 s to an estimated 500 acres in 2014 (Lobo, personal communication). Similar trends are being observed in other pitayaha-producing states like Florida and Hawaii. However, because of limited information about this crop, growers are making decisions under high uncertainty, which makes pitahaya cultivation an expensive and risky venture. The results of this study will help to streamline studies in the future to determine which SCREC accessions will be the most profitable for California growers. It will also allow growers from other regions to choose genetically distinct pitahaya accessions from the germplasm collection to investigate how different accessions would perform in their growing environments.

\section{Literature Cited}

Ashworh, V.E.T.M. and M.T. Clegg. 2003. Microsatellite markers in avocado (Persea americana Mill.): Genealogical relationships among cultivated avocado genotypes. J. Hered. 94:407-415.

Bauer, R. 2003. A synopsis of the tribe Hylocereeae F. Buxb. Cac. Systm. Init. 17:3-63.
Benham, J., J.U. Jeung, M. Jasieniuk, V. Kanazin, and T. Blake. 1999. Genographer: A graphical tool for automated fluorescent AFLP and microsatellite analysis. J. of Ag. Gen. 4:1-3.

Britton, N.L. and J.N. Rose. 1920. The cactaceae: Descriptions and illustrations of plants of the cutus family. Vol. 2. The Carnegie Institute of Washington, WA.

Castillo-Martinez, R., M. Libera-Muñoz, and G.J. Marquez-Guzman. 2005. Morphological characterization and sexual compatibility of five Pitahayas (Hylocereus undatus) genotypes. Agrociencia 39:183-194.

Crane, J. and C.F. Balerdi. 2009. Pitaya growing in the Florida home landscape. Univ. of Florida IFAS Exten. HS 1068.

Dimitri, J. 1999. Pitaya (Hylocereus undatus), a potential new crop for Australia. The Australian New Crops Newsletter 29:16.3.

Instituto Nicaraguense de Reforma Agraria. 1994. Guia Tecnologica Para la Produccion de Pitahaya. 2da Edicion, Abril de 1994. Gobierno de Nicaragua, Proyecto Desarrollo de la Produccion Agricola en la Zona de la Meseta CEEALA 86/30. San Marcos, Carazo, Nicaragua.

International Union for the Protection of New Varieties of Plants (UPOV). 2011. Dragon fruit: Guidelines for the conduct of tests for distinctness, uniformity and stability. UPOV, Geneva, Switzerland. p. 10-20.

Joubert, R. 2012. Riding the Dragon. Farmers Weekly Magazine, Republic of South Africa. 8 June 2012.

Komang, D.J. 2010. Morphology and physiology of Pitaya and its future prospects in Indonesia. Crop Agro. Vol. 3, No. 1. January.

Le Bellec, F., F. Vaillant, and E. Imbert. 2006. Pitahaya (Hylocereus sp.): A new fruit crop, a market with a future. Fruits 61:237-250.

Legaria Solano, J.P., M.E. Alvarado Cano, and R.G. Hernandez. 2005. Genetic diversity of Pitahaya (Hylocereus undatus Haworth, Britton and Rose). Rev. Fitot. Mex. 28:179-185.

Lobo, R. 2007. Pitahaya (Dragon gruit) Research \& Production in California. UC Small Farm Program Specialty Crops Conference. Davis, CA. 12 Dec. 2007. <http://cesandiego.ucdavis.edu/ files/55065.pdf>.

Luders, L. and G. McMahon. 2006. The pitaya or Dragon fruit (Hylocereus undatus). Agnote Number: D42, May 2006. Department of
Primary Industry, Fisheries and Mines, Northern Territory Government.

Merten, S. 2003. A review of Hylocereus production in the United States. J of the Prof. Assoc. for Cactus Dev. 5:98-105 (JPACD).

Morgan, R.V. 1953. Selenicereus magalanthus (Schuman) Moran. Gen. Herb. 8:325.

Nobel, P.S. and E. De LaBarrera. 2004. $\mathrm{CO}_{2}$ uptake by the cultivated hemiepiphytic cactus Hylocereus undatus. Ann. Appl. Biol. 144:1-8.

Ortiz, Y.D.H. and J.A.S. Carrillo. 2012. Pitahaya (Hylocereus spp.) A short review. Comm. Sci. 3:220-237.

Plume, O., C.K. Sraub, N. Tel-Zur, A. Cisneros, B. Schneider, and F.F. Doyle. 2013. Testing a hypothesis of intergeneric alloploidy in vine cacti (Cactaceae:Hylocereea). Syst. Bot. 38:737-751.

Raveh, E., A. Nerd, and Y. Mizrahi. 1998. Responses of two hemi-epiphytic fruit crop cacti to different degrees of shade. Sci. Hort. 73:151-164.

Raveh, E., J. Weiss, A. Nerd, and Y. Mizrahi. 1993. Pitayas (Genus Hylocereus): A new fruit crop for the Negev Desert of Israel, p. 491-495. In: Janick, J. and J.E. Simons (eds.). New crops. Wiley, New York, NY.

Swofford, D.L. 2002. PAUP*. Phylogenetic analysis using parsimony (*and other methods), version 4.0b10 (Alvitec). Sinauer Associates, Sunderland, MA.

Tao, J., G. Qiao, X.P. Wen, G.L. Gao, T. Liu, Z.J. Peng, Y.Q. Cai, N. Chen, F.X. Yan, and B.X. Zhang. 2014. Characterization of genetic relationships of dragon fruit accessions (Hylocereus sp.) by morphological traits and ISSR markers. Sci. Hort. 170:82-88.

Tel-Zur, N., S. Abbo, D. Bar-Zvi, and Y. Mizrahi. 2004. Clone identification and genetic relationship among vine cacti from the genera Hylocereus and Selenicereus based on RAPD analysis. Sci. Hort. 100:279-289.

Thomson, P. 2002. Pitahaya (Hylocereus species): A promising new fruit crop for southern California. Bonsall Publications, Bonsall, CA.

Valdivia, E. 2000. Pitahaya: A fruit for the diligent. The Fruit Gard. 32:12-13.

Vos, P., R. Hogers, M. Bleeker, M. Reijans, T. van de Lee, M. Hornes, A. Frijters, J. Pot, J. Peleman, M. Kuiper, and M. Zabeau. 1995. AFLP: A new technique for DNA fingerprinting. Nucleic Acids Res. 23:4407-4414. 